\title{
Resultados a mediano plazo del trasplante osteocondral autólogo en las lesiones del cartílago patelar
}

\author{
Medium-term results of autologous osteochondral \\ transplantation in patellar cartilage injuries \\ Alonso-Polo B, * Sobrón FB, ${ }^{\ddagger}$ Rodríguez-Guerrero J* \\ Hospital Universitario Infanta Cristina. Parla, España.
}

RESUMEN. Introducción: El cartílago rotuliano está sometido a altas demandas mecánicas y es una localización muy frecuente de lesiones. No existe consenso en cuanto a su tratamiento. El trasplante osteocondral autólogo puede ser una alternativa para los defectos condrales de pequeño o mediano tamaño con resultados variables en la bibliografía. Nuestro objetivo es analizar los resultados funcionales a mediano plazo de los pacientes con lesiones del cartílago patelar grado III-IV de la International Cartilage Repair Society (ICRS) tratados en nuestro centro. Material y métodos: Estudio retrospectivo con pacientes menores de 55 años, con un defecto condral patelar sintomático, diámetro menor de $2 \mathrm{~cm}$, grado III-IV de la ICRS tratados mediante trasplante osteocondral autólogo entre los años 2012 y 2018. Se evalúan las siguientes variables: edad, sexo, tamaño de la lesión, faceta afectada, número de cilindros trasplantados, escala de Kujala pre y postoperatoria y escala visual analógica del dolor (EVA) pre y postoperatoria. Resultados: Integramos en nuestro estudio 11 pacientes. La mediana de edad fue de 47 años. La mediana del diámetro del defecto condral $1.3 \mathrm{~cm}$. La mediana de seguimiento 3.9 años (1.84-5.58 años). La mediana del cuestionario Kujala preoperatoria es 33 y asciende a 89 al final del seguimiento ( $p$ $=0.003$ ). La mediana de la puntuación EVA preoperatoria es nueve y dos al final del seguimiento $(\mathrm{p}=0.003)$. Conclusión: El trasplante osteocondral autólogo generó una importante mejora subjetiva de la función y del dolor.

Palabras clave: Patela, cartílago, trasplante, osteocondral, defecto.
ABSTRACT. Introduction: Patellar cartilage is subject to high mechanical requests and is a very frequent location of injuries. There is no consensus on their treatment. Autologous osteochondral transplantation may be an alternative to small to medium-sized condral defects with varying results in the literature. Our goal is to analyze the medium-term functional outcomes of patients with grade III-IV patellar cartilage injuries from the International Cartilage Repair Society (ICRS) treated at our facility. Material and methods: Retrospective study in patients under 55 years of age, with a symptomatic patellar condral defect, diameter less than $2 \mathrm{~cm}$, grade III-IV of ICRS treated by autologous osteochondral transplant between 2012 and 2018. The following variables are evaluated: age, sex, injury size, affected facet, number of transplanted cylinders, preand postoperative Kujala score, and pre- and postoperative analog visual pain scale (EVA). Results: Eleven patients integrated into our study. The median age was 47 years. The median diameter of the condral defect $1.3 \mathrm{~cm}$. Median follow-up 3.9 years (1.84-5.58 years). The median of the preoperative Kujala questionnaire is 33 and is 89 at the end of the follow-up $(\mathrm{p}=0.003)$. The median of the preoperative EVA score is 9 and 2 at the end of the follow-up ( $p=0.003)$. Conclusion: Autologous osteochondral transplantation generated a significant subjective improvement in function and pain.

Keywords: Patella, cartilage, transplantation, osteochondral, defect.

\section{Nivel de evidencia: IV}

* Cirugía Ortopédica y Traumatología, Hospital Universitario Infanta Cristina. Parla, España.

‡ Cirugía Ortopédica y Traumatología, Hospital General Universitario Gregorio Marañón. Madrid, España.

Dirección para correspondencia:

Dra. Berta Alonso-Polo.

Av. 9 de Junio, 2, CP 28981, Parla, Madrid, España.

E-mail: mariaberta.alonso@salud.madrid.org 


\section{Introducción}

El cartílago patelar está sometido a altas demandas mecánicas, soporta cargas de hasta 6.5 veces el peso del cuerpo durante las actividades cotidianas. ${ }^{1}$ Después del cóndilo femoral medial es la localización más frecuente de lesiones condrales hasta $34.6 \%$ en algunas series. ${ }^{2}$ Los defectos condrales en superficies de carga pueden manifestarse como dolor, inflamación o chasquidos y pueden originar una degeneración precoz y artrosis secundaria.

El tratamiento de estas lesiones supone un reto con diversas técnicas descritas y sin un consenso establecido. Existen diversos factores que influyen en la toma de decisiones como el tamaño y localización de la lesión, pero también factores dependientes del paciente como la edad, el nivel de actividad deportiva o desviaciones en los miembros inferiores. ${ }^{4}$

El trasplante osteocondral autólogo puede ser una alternativa para pacientes activos con defectos condrales grado III-IV de la International Cartilage Repair Society (ICRS) de pequeño o mediano tamaño $\left(2-4 \mathrm{~cm}^{2}\right)$. Consiste en la extracción de uno o más cilindros de injerto osteocondral, de un sitio donante de mínima carga de la misma articulación y su impactación en la zona de la lesión condral con el objetivo de restaurar la superficie articular. ${ }^{4}$

Es un procedimiento técnicamente demandante con resultados variables. En la bibliografía existen estudios que muestran resultados muy satisfactorios a largo plazo, $, 3,6$ mientras que otras publicaciones reportan hasta 100\% de fracasos.

El objetivo de este estudio es analizar los resultados funcionales a mediano plazo de los pacientes con lesiones del cartílago patelar grado III-IV ICRS tratados en nuestro centro mediante trasplante osteocondral autólogo.

Material y métodos

Presentamos un estudio retrospectivo que incluye pacientes menores de 55 años, diagnosticados de defecto condral patelar sintomático, con un diámetro menor de $2 \mathrm{~cm}$, grado III-IV de la ICRS que fueron tratados mediante trasplante osteocondral autólogo en nuestro centro entre los años 2012 y 2018. Se excluyen aquellos pacientes con lesiones coexistentes en la rodilla intervenida como lesiones ligamentosas, artrosis o con inestabilidad femoropatelar asociadas.

Las lesiones condrales fueron diagnosticadas y caracterizadas de manera preoperatoria mediante resonancia magnética nuclear como parte del estudio de los pacientes con síndrome de dolor anterior de rodilla refractario al tratamiento conservador.

Todas las intervenciones quirúrgicas fueron realizadas por un mismo cirujano. Se utilizó un abordaje parapatelar longitudinal medial con eversión de la rótula para visualizar la superficie articular. Se miden los diámetros de la lesión con una regla y mediante la utilización de una cureta se consiguen unos bordes del cartílago perilesional circulares y perpendiculares. En función del tamaño de la lesión, se decide el número de cilindros de injerto osteocondral que va a precisarse y su diámetro más adecuado. Como zona donante se utiliza la tróclea femoral lateral superior, que es una zona sin carga. Se obtienen los cilindros osteocondrales mediante la utilización de un extractor específico, colocado de manera perpendicular a la superficie articular donante. También se empleó material específico para perforar la zona receptora rotuliana (Figura 1). Los cilindros fueron impactados hasta alcanzar el nivel de la superficie articular (Figura 2).

Se permite la movilización activa de la rodilla desde el postoperatorio inmediato, el rango de movilidad se va aumentando progresivamente según la tolerancia del paciente. La carga sobre el miembro inferior intervenido se inicia durante la tercera semana postoperatoria. No se autoriza correr hasta el cuarto mes y los deportes de alta demanda funcional se reinician a partir del sexto mes. ${ }^{8}$

La recopilación de datos se realiza mediante revisión de las historias clínicas a través del soporte informático. Las variables recogidas fueron edad, sexo, fecha de la intervención, tamaño de la lesión, faceta afectada, número de cilindros trasplantados, escala de Kujala pre y postoperatoria y escala visual analógica del dolor (EVA) pre y postoperatoria. Tras la realización de la estadística descriptiva se lleva a cabo el contraste de hipótesis utilizando pruebas no paramétricas debido al tamaño muestral. Se emplea el test de Wilcoxon para muestras relacionadas, estableciéndose como estadísticamente significativo $\mathrm{p}<0.05$.

El protocolo del presente estudio fue aprobado por la Comisión de Ética de Investigación Clínica de nuestra institución. Los autores no han recibido ninguna financiación para la realización del estudio.

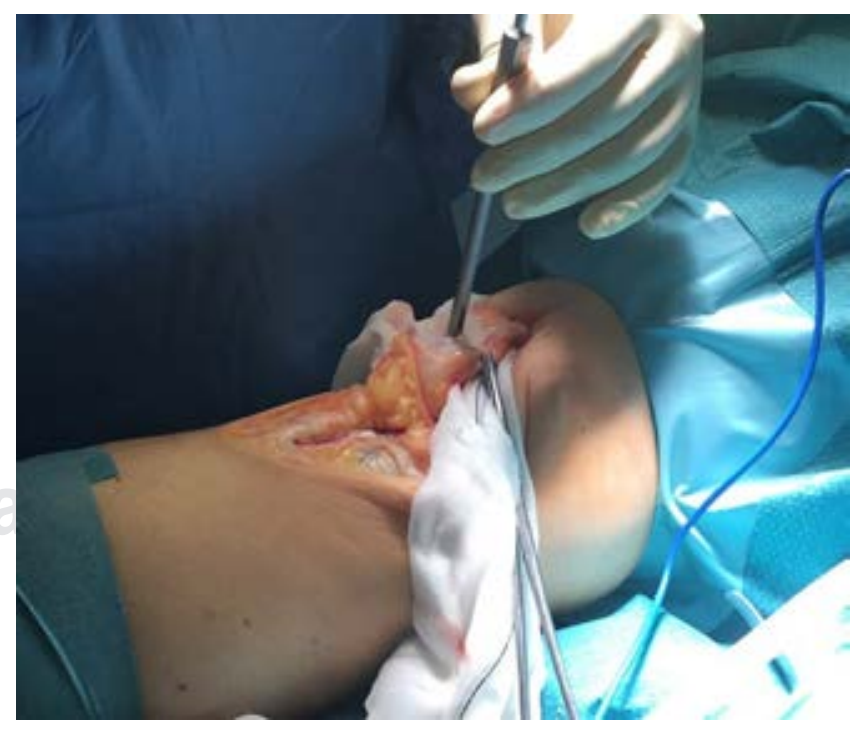

Figura 1: Imagen intraoperatoria. Mediante un abordaje parapatelar longitudinal medial con eversión de la rótula se visualiza la superficie articular. Se utilizan extractores específicos del diámetro adecuado, colocados de manera perpendicular a la superficie articular para obtener los cilindros osteocondrales y para perforar la zona receptora rotuliana. 


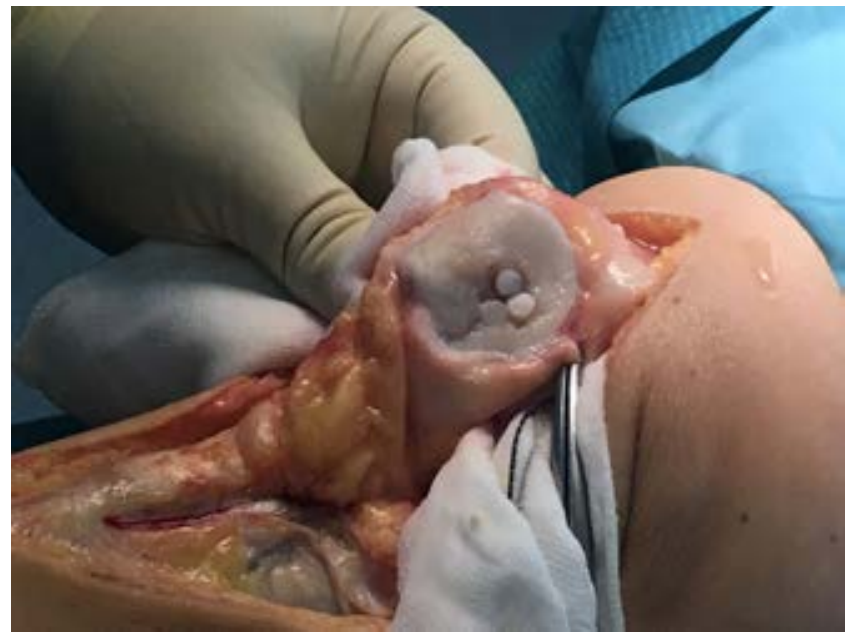

Figura 2: Imagen intraoperatoria. En función del tamaño de la lesión se decide el número y el diámetro de los cilindros osteocondrales precisados. Dichos cilindros deben impactarse hasta alcanzar la superficie articular patelar.

\section{Resultados}

Se incluyeron en nuestro estudio 11 pacientes, siete mujeres y cuatro hombres. La mediana de edad en el momento de la cirugía es 47 años (rango intercuartílico RIC: 31-51.5 años). La mediana de seguimiento es 3.9 años (RIC: 1.845.58 años). La mediana del diámetro del defecto condral es $1.3 \mathrm{~cm}$ (RIC: 1.1-1.5 cm). En ocho pacientes (72\%) la lesión se presentaba en la faceta medial, en un paciente (9\%) la faceta afectada era la lateral y en dos pacientes (18.2\%) estaban afectadas ambas facetas (Tabla 1).

En $82 \%$ de los pacientes (nueve) se implanta un número de cilindros osteocondrales igual o menor de tres. Todos los cilindros fueron realizados con una longitud de $10 \mathrm{~mm}$. La mediana del cuestionario Kujala preoperatoria es 33 (RIC: 22-34) y asciende a 89 (RIC: 54-96) al final del seguimiento $(\mathrm{p}=0.003)$. La mediana de la puntuación EVA preoperatoria es nueve (RIC: 7-10) y dos (RIC: 0-8) al final del seguimiento $(\mathrm{p}=0.003)$.

En ningún caso se realizaron técnicas asociadas para el tratamiento de la inestabilidad femoropatelar.

\section{Discusión}

El tratamiento de las lesiones del cartílago patelar supone un reto debido a la complejidad y a las peculiaridades anatómicas de esta articulación. Existen diversas opciones quirúrgicas como las microfracturas, el trasplante osteocondral autólogo o alogénico o las terapias regenerativas con implante de condrocitos autólogos, que podrían emplearse en función de factores dependientes del paciente y de la lesión. No existe ningún consenso establecido debido a la escasa evidencia de los estudios publicados. ${ }^{4}$

Barbieri propone un algoritmo de tratamiento en el que recomienda el trasplante osteocondral autólogo en las lesiones de espesor completo con afectación del hueso subcondral de menos de $2 \mathrm{~cm}^{2}{ }^{9}$ La aplicación de esta técnica en lesiones de mayor tamaño estaría limitada por la disponibilidad del sitio donante, ${ }^{4}$ además en algunos estudios se sugieren mejores resultados con el trasplante osteocondral autólogo en las lesiones con un diámetro menor de $2.5 \mathrm{~cm} .{ }^{10}$ Para las lesiones mayores de $2 \mathrm{~cm}^{2}$ podría emplearse el trasplante osteocondral alogénico o las terapias regenerativas. $^{9}$

Existen varias publicaciones que, al igual que nuestro estudio, avalan el trasplante osteocondral autólogo en la rótula con buenos resultados funcionales a corto, mediano y largo plazo. 3,5,6,8,10,11,12 Figueroa y colaboradores realizan un estudio prospectivo con un seguimiento medio de tres años, que incluye 10 pacientes con lesiones osteocondrales patelares grado IV de ICRS, tratados con trasplante osteocondral autólogo y encuentran una mejora estadísticamente significativa de la escala Lysholm, con 100\% de resultados satisfactorios, $60 \%$ buenos y $40 \%$ excelentes. ${ }^{12,13}$ Nuestro estudio muestra una mejora subjetiva de la función y el dolor con un incremento significativo de la escala funcional Kujala y de la puntuación EVA a mediano plazo.

Otros trabajos muestran resultados menos satisfactorios tras la realización de esta técnica. Bentley y su equipo llevan a cabo un estudio prospectivo en el que incluyen cinco pacientes con trasplante osteocondral autólogo patelar, los resultados funcionales son regulares y en un plazo máximo

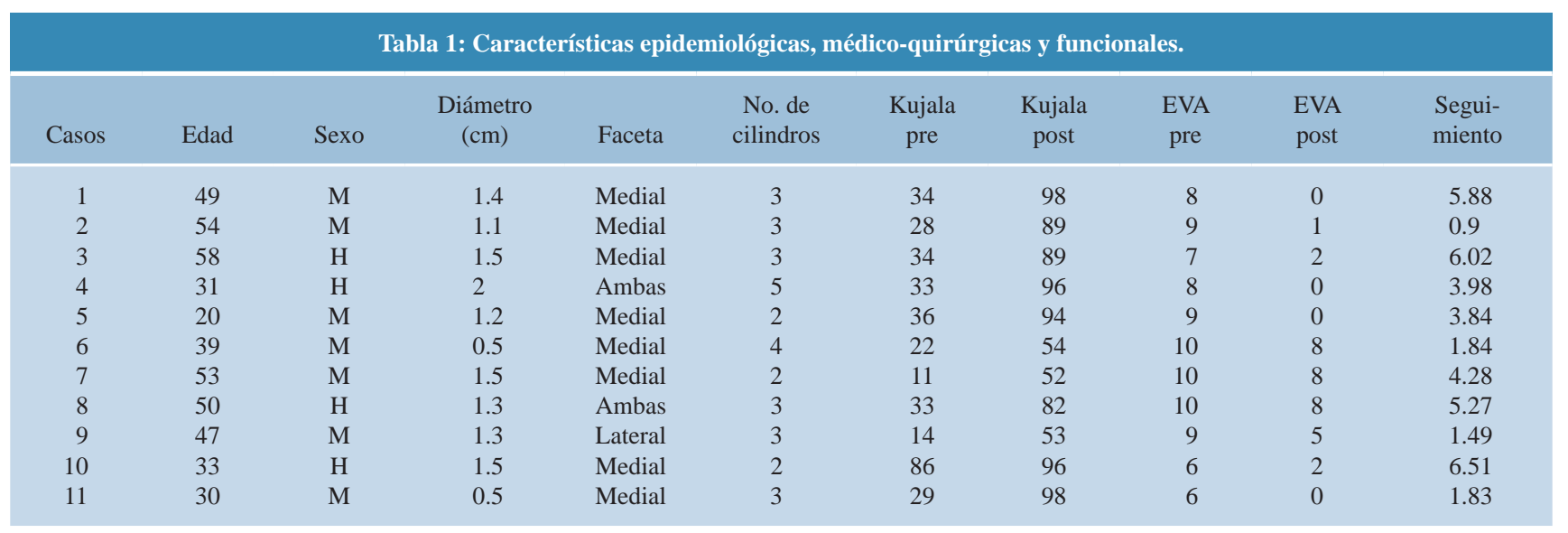


de dos años todos precisan de una nueva cirugía. ${ }^{7}$ Para alcanzar resultados satisfactorios con el trasplante osteocondral autólogo es fundamental una buena indicación quirúrgica y tratar la patología femoropatelar concomitante antes de abordar la lesión osteocondral. Nho y colaboradores refieren que los pacientes con mal alineamiento femoropatelar tienen peores resultados funcionales tras el trasplante osteocondral patelar. ${ }^{11}$ En el presente estudio se excluyen los pacientes con otras alteraciones anatómicas concomitantes con el objetivo de evitar sesgos.

En la bibliografía también se sugiere que los resultados del trasplante osteocondral autólogo en los defectos del cartílago patelar son inferiores respecto a los obtenidos por esta misma técnica en otras localizaciones de la rodilla. En la serie de Hangody se obtiene $74 \%$ de buenos resultados a los 10 años en lesiones patelares, frente a 92\% de buenos resultados en lesiones condrales del cóndilo femoral medial. ${ }^{3}$ Esto podría estar relacionado con la dificultad para conseguir una adecuada congruencia articular en la rótula y por la discrepancia entre el grosor del cartílago patelar, que puede alcanzar un grosor de hasta $10 \mathrm{~mm}$ y el del sitio donante, que suele ser menor de $2 \mathrm{~mm} .{ }^{4}$ Estas peculiaridades podrían someter a los cilindros trasplantados a elevadas cargas cíclicas que justificarían que el rendimiento del trasplante osteocondral autólogo patelar fuera menor que en otras localizaciones. ${ }^{4}$

Hangody señala como límite superior recomendado para la utilización del trasplante osteocondral autólogo la edad de 50 años, basándose en la disminución de la capacidad biológica de reparación. ${ }^{3}$ En nuestra serie incluimos pacientes de hasta 55 años, sin signos de artrosis u otras lesiones concomitantes con resultados muy satisfactorios.

Nuestro estudio presenta varias limitaciones. Primero, el número de pacientes que limita el poder estadístico del estudio y segundo que se trata de un estudio retrospectivo. Tercero, carece de grupo control para comparar esta técnica con otra. Cuarto, los resultados están basados en escalas subjetivas sin analizar parámetros objetivos.

En conclusión, el trasplante osteocondral autólogo es una técnica válida para el tratamiento de los defectos condrales patelares grado III-IV de la ICRS sintomáticos de menos de $2 \mathrm{~cm}$ de diámetro con una importante mejora subjetiva de la función y del dolor.

\section{Bibliografía}

1. Huberti HH, Hayes WC. Patellofemoral contact pressures. The influence of q-angle and tendofemoral contact. J Bone Joint Surg Am. 1984; 66(5): 715-24.

2. Figueroa D, Calvo R, Vaisman A, Carrasco MA, Moraga C, delgado I. Knee chondral lesions: incidence and correlation between arthroscopic and magnetic resonance findings. Arthroscopy. 2007; 23(3): 312-5.

3. Hangody L, Vásárhelyi G, Hangody LR, et al. Autologous osteochondral grafting--technique and long-term results. Injury. 2008; 39 Suppl 1: S32-9.

4. Díaz P, Figueroa D. Trasplante osteocondral autólogo en rodilla: ¿dónde están las limitaciones? Rev Esp Artrosc Cir Articul. 2018; 25(Supl. 1): 46-58.

5. Hangody L, Füles P. Autologous osteochondral mosaicplasty for the treatment of full-thickness defects of weight-bearing joints: ten years of experimental and clinical experience. J Bone Joint Surg Am. 2003; 85(Suppl. 2): 32-9.

6. Jakob RP, Franz T, Gautier E, Mainil-Varlet P. Autologous osteochondral grafting in the knee: indication, results, and reflections. Clin Orthop Relat Res. 2002; (401): 170-84.

7. Bentley G, Biant LC, Carrington RW, et al. A prospective, randomised comparison of autologous chondrocyte implantation versus mosaicplasty for osteochondral defects in the knee. J Bone Joint Surg Br. 2003; 85(2): 223-30.

8. Astur DC, Bernardes A, Castro S, et al. Functional outcomes after patellar autologous osteochondral transplantation. Knee Surg Sports Traumatol Arthrosc. 2017; 25(10): 3084-91.

9. Mestriner AB, Ackermann J, Gomoll AH. Patellofemoral cartilage repair. Curr Rev Musculoskelet Med. 2018; 11: 188-200.

10. Astur DC, Arliani GG, Binz M, et al. Autologous osteochondral transplantation for treating patellar chondral injuries: evaluation, treatment, and outcomes of a two-year follow-up study. J Bone Joint Surg Am. 2014; 96(10): 816-23.

11. Nho SJ, Foo LF, Green DM, et al. Magnetic resonance imaging and clinical evaluation of patellar resurfacing with press-fit osteochondral autograft plugs. Am J Sports Med. 2008; 36(6): 1101-9.

12. Emre TY, Atbasi Z, Demircioglu DT, Uzun M, Kose O. Autologous osteochondral transplantation (mosaicplasty) in articular cartilage defects of the patellofemoral joint: retrospective analysis of 33 cases. Musculoskelet Surg. 2017; 101(2): 133-8.

13. Figueroa D, Meleán P, Calvo R, Gili F, Zilleruelo N, Vaisman A. Osteochondral autografts in full thickness patella cartilage lesions. Knee. 2011; 18(4): 220-3. 